\title{
LA COSMOVISIÓN PEIRCEANA COMO HERRAMIENTA EPISTEMOLÓGICA PARA LEER LA FÍSICA
}

\author{
Lucía Velasco Víllarreal
}

\begin{abstract}
The purpose of the article below is to show how some cognitive prejudices cristallize human knowledge therefore not permitting It to evolve. To demonstrate this I will explain in sorne detaill Ch. S. Peirce article "The architecture of theories". In this article Peirce attract our attention in order to make the different sciences communicate again. He did this by studying the law concept of different sciences and abstracting a general definition. This is used to widen the law epistemologicl conception, by means of taking information from general to the particular and viceversa.
\end{abstract}

\section{RESUMEN}

El propósito del presente artículo es mostrar cómo se podrían resquebrajar algunos prejuicios cognitivos que cristalizan el conocimiento humano y no lo dejan evolucionar. Para ello, presentaré con algún detalle el artículo de Ch. S. Peirce "La arquitectura de las teorías". En este artículo, Peirce llama la atención en volver a comunicar las diferentes ciencias, su hilo conductor es el concepto de ley, el cual analiza en diferentes ciencias para abstraer una definición general, que sirve para ampliar la concepción epistemológica en la medida en que lleva información de lo general a lo particular y viceversa.

Palabras clave: conocimiento, ley, evolución, hábito, continuidad, interdisciplinariedad.

El análisis del conocimiento humano se puede abordar desde infinitas rutas. En este artículo, se quieren señalar dos aspectos: 1. Este análisis revive discusiones del pasado, como la discusión entre realistas y nominalistas, en la medida, en que se pregunta si lo que conocemos está en la mente o, fuera de ella. La pregunta base es si el conocimiento se adquiere por medio de la acción de la mente o por medio de la acción de los sentidos. 2. El reconocimiento de algunos prejuicios cognitivos; por ejemplo, resulta sorprendente ver el arraigo de ciertas ideas o principios rectores del pensamiento, tales como la geometría euclidiana, el dualismo cartesiano y la dualidad verdadero-falso. Todas estas, ideas que favorecen una concepción determinista del mundo que deja por fuera una inmensa gama de posibilidades. En este caso no se sabría si darle la razón a Platón cuando dice que, desde el momento en que nacemos, traemos ya todo el conocimiento, y que sólo es cuestión de tener una buena partera para poder parir las ideas. O se trata más bien de que la concepción de educación de los países llamados del tercer mundo, no favorece el desarrollo intelectual de los niños, sino que castra cualquier acto creativo de su parte. De esta manera se estarían formando prejuicios que la misma tradición

\footnotetext{
“Profesora catedrática de la Universidad Pedagógica Nacional. Ivelasco2002 @ yahoo.es 
educativa va incorporando en la cabeza de los estudiantes, en la medida en que no se muestran otras opciones geométricas, metafísicas y lógicas.

Por lo tanto, el propósito de este artículo no es indicar una polémica acerca de los prejuicios cognitivos, sino mostrar concepciones alternativas al mecanicismo y al determinismo moderno, arraigado no sólo en el sentido común de nuestros estudiantes, sino de nosotros mismos. Charles Sanders Peirce (1839-1914) nos hace caer en cuenta que el conocimiento está en constante evolución. Grosso modo, lo que Peirce propone es examinar el conocimiento humano, pero no en una única ciencia, dado que lo que se diría allí sería muy pobre. Él recurre a varias ciencias para ver de qué manera se aborda y cómo se relaciona con otros, el concepto más común. Ese concepto no es otro que el de ley. Algunas veces se considera a la ciencia como un pequeño feudo que cierra sus puertas al exterior, pero habría más riqueza cuando se consideran aspectos tales como: el devenir de generalidades a particulares, la interdisciplinariedad y la transdisciplinariedad, y el azar y la evolución, entre otros aspectos. Los términos generales, guardando las debidas proporciones, son como los mapas que representan una región, aunque ayuden a orientar, no pueden dar datos especiales del territorio. Pero cuando se recorre el territorio habría un mejor desenvolvimiento, si se tiene en cuenta dicho mapa. Es decir, para definir un concepto en una ciencia particular es importante tener una noción amplia de su definición general.

A continuación, se quiere retomar uno de los artículos de Peirce, "La arquitectura de las teorías", y presentar con cierto detalle la propuesta que se indicó en el párrafo anterior, la cual sirve para hacer una lectura epistemológica en cualquier ciencia. Aproximadamente, desde los años 60, la cosmovisión peirceana se ha ido comprendiendo, gracias al rescate de sus papers, por parte de algunos filósofos y matemáticos de cierta prestancia académica. En el tercer período, como denomina Apel uno de los periodos de producción intelectual de Peirce, él escribe una serie de cinco artículos en El Monista, intentando unir nuevamente lógica y metafísica. Este contexto particular nos da herramientas para combatir prejuicios que adquirimos culturalmente: "El tercer periodo engloba aquellos años (de 1883 a 1893 o a 1902) en los que Peirce -especialmente, tras su traslado a Milford (Pennsylvania) - trabajó en solitario en diversos estudios sobre lógica y metafísica, y concluyó aproximadamente en 1901/02, la arquitectónica definitiva de su sistema filosófico" (Apel, 1997).

El primer artículo de esta serie y del único que se hará mención aquí, se titula, "La arquitectura de la teorías, en éste está condensada la intención de la serie completa; resquebrajar la parte del sistema cartesiano y del sistema euclidiano, que reduce la mente humana a un sistema cerrado. Peirce aborda este problema como un problema propio de la filosofía, pero como se indicará más adelante, se puede ampliar a otros dominios del saber. Inicialmente, Peirce no está conforme con el método que hasta ahora ha llevado la filosofía, pues considera que las ideas filosóficas se presentan como pensamientos accidentales y no como parte de una evolución histórica. Por esta razón, compara las ideas filosóficas con una construcción horrible. Si bien es cierto que una casa se construye con diferentes materiales, se debe buscar los materiales más adecuados para construir una casa sólida y armoniosa. Respecto del conocimiento, también se deberían buscar los conceptos adecuados para construir un sistema sólido y armonioso, y en constante evolución, pues, de lo contrario se le daría la razón a Peirce cuando dice: “(...) así son las ideas filosóficas sumamente interesantes e instructivas, pero carentes de solidez." (C.P. 6.7) 1 .

\footnotetext{
${ }^{1}$ Las traducciones del presente artículo son de Lacia Velasco. 
Antes de entrar en materia, vale la pena mencionar que una de las características más importantes del pensamiento peirceano, es la relación triádica (1-2-3) que desde el punto de vista lógico abre la dualidad verdadero-falso, introduciendo lo posible. Hay una triada fundamental definida de manera general y flexible (como se verá más adelante), susceptible de ser aplicada a casos particulares. La relación triádica va formando un tejido que no sólo retoma hilos científicos, sino políticos y culturales. Por tal razón, la discusión internalismo-externalismo, dentro de la cosmovisión peirceana, pierde importancia. No se trata de analizar de qué depende el progreso científico, si de la ciencia misma o de aspectos ajenos a ella. El interés de Peirce es establecer relaciones entre unas cosas y otras, entre unos conceptos y otros, entre cosas y conceptos, y entre relaciones mismas. Y así como pierde importancia la discusión internalismo-externalismo, también lo pierde la de objetivo-subjetivo. Aquí lo importante son las relaciones. Sólo que en este artículo en particular, Peirce analiza ciertas relaciones en diferentes ciencias.

Lo que se debe hacer, según Peirce, es: "realizar un examen riguroso del conocimiento humano, tomar nota de todas las ideas valiosas en cada rama de las ciencias, observar respecto a qué han sido prósperas en cada ciencia y dónde se han abandonado (...), bajo la luz de un conocimiento completo se puede obtener el material aprovechable para una teoría filosófica (...)" (C.P. 6.9) ${ }^{2}$. Dentro de la concepción peirceana, se puede entender por "conocimiento completo" no algo acabado, sino, más bien, un contexto general en el que se debe aclarar el sentido de los conceptos. El cual no puede tener otro método, que el método sintético.

Peirce inicia el examen, en la dinámica ${ }^{3}$, con la ley de conservación de la energía, e indica la postura de Galileo, respecto a la noción de ley, y su interés por il lume natural: "Él siempre asumió que la teoría verdadera se encontraría por estar en una simple y natural unidad" (C.P. 6.10), lo cual se puede observar cuando los físicos aplican la idea de líneas rectas, no sólo a la caída de los cuerpos por acción de la gravedad, sino al movimiento de la luz, por la importancia que tienen las líneas rectas en la geometría euclidiana: "Así, nuestra mente ha sido formada bajo la influencia de fenómenos gobernados por las leyes mecánicas, ciertas concepciones entran en esas leyes que vienen implantadas en nuestra mente, y así nosotros prontamente suponemos lo que las leyes son" (C.P. 6.10).

Gracias a las citas anteriores, se observa, por una parte, que las leyes son naturales y simples y, por otra, que la concepción de ley es realista en la medida en que llegamos a las leyes, porque ellas están inmersas en la búsqueda.

El siglo XVII dio origen a la física, no sólo como disciplina independiente, sino como paradigma científico. En esta época se consolidó un malestar y una alternativa respecto al pensamiento aristotélico. Francis Bacon y René Descartes, indicaron el camino a seguir ${ }^{4}$, aunque no pasaron a la historia con el nombre de científicos, como sí lo hicieron Galileo, Newton y Huygens, entre otros. Esta nueva ciencia explica todos los fenómenos por medio de atracciones y repulsiones entre cuerpos, los cuales se pueden reducir a puntos, ya sea, que se considere un electrón o un planeta. Esto se puede hacer porque a los cuerpos se les puede determinar su centro de masa. Esta propuesta mecánica llega a

\footnotetext{
${ }^{2}$ La numeración canónica de Peirce escomo se presenta a continuación; por tal motivo, no se escribirá el autor y el año, como recomienda la Revista TEA.

3 Actualmente denominada física.

${ }^{4}$ Se dice esto pero no hay que desconocer el trabajo de Roger Bacon,Rober Grosseteste, y Guillermo de Occam, entre otros.
} 
lugares impensables: "La vieja noción de que el calor consiste en una agitación de Corpúsculos ahora era aplicada a la explicación de la propiedad principal de los gases"

(C.P. 6.11). Thompson, kelvin, Rankin, John Bernoulli, Avogadro y Crookes, iniciaron su de trabajo distanciándose de la noción de "acción a distancia"Newton, acercándose a la idea del plenum cartesiano, pero la teoría del calor vuelve a los principios mecánicos, con la mecánica estadística. Sin embargo, y a pesar de ello, hay otros fenómenos que requieren hipótesis adicionales, tales como: “(...) la difusión y viscosidad de los gases (...) y la radiometria" (C.P. 6.11). Con estos nuevos requerimientos, ya no se pueden considerar las leyes, de manera simple, inclusive, surge la pregunta: ¿las hipótesis son ensayos casuales o conviene a los fenómenos y de ello se tendría que ocupar la fisicomatemática? Las teorías podrían ser millones. A este respecto, Peirce dice: "Nosotros tenemos un pequeño prospecto para hacer más sólida la sumatoria de temas de nuestro tiempo" (C.P. 6.11). Ese prospecto no es otro que el método sintético, que más que explicarse, se muestra.

El examen del conocimiento humano, cuando pasa por la física, muestra que la noción de ley arroja datos importantes, entre ellos, que cuando se conocen las condiciones iniciales de una partícula, su movimiento se puede determinar con precisión, gracias al principio de causalidad. De esta manera se explican muchos fenómenos, pero no todos los fenómenos caen bajo sus dominios; hay muchos casos en los que se necesitan hipótesis subsidiarias o hipótesis que no se contemplaban en latearía. Parlo tanto, será necesario ir a otras disciplinas y estudiar el comportamiento de sus leyes para poder consolidar una noción general de ley, con el propósito de volver nuevamente a la ciencia particular y hacer, así, un análisis epistemológico con más elementos cognitivos.

Posteriormente, Peirce pasa a una disciplina que pueda suplir las falencias que se encontraron en la física, y la biología, ya que la uniformidad puede ser esa clase de hechos que se deben considerar: "Ahora el único sentido posible de considerar las leyes de la naturaleza y su uniformidad en general es suponer los resultados de la evolución" (C.P. 6.13). Esto implica dos cosas: que las leyes ya no se deben considerar como absolutas, sino en evolución, y con una dirección específica del tiempo, situaciones contrarias a lo que ocurría en la física, pues allí, por el carácter determinista de las leyes, éstas se debían considerar como absolutas y reversibles. La evolución considera elementos de indeterminación, espontaneidad y azar en la naturaleza.

Peirce dice que hablar de la evolución en términos mecanicistas es un absurdo. Herbert Spencer cree poder hacerlo, pero se encuentra, parlo menos, con cuatro inconvenientes:

1) El principio de evolución no requiere causas extrañas.

2) La ley se debe considerar como resultado de la evolución.

3) Las leyes deterministas nunca pueden producir heterogeneidad de la homogeneidad.

4) La ley de conservación de la energía es equivalente a la reversibilidad, consideración que impediría un desarrollo.

A propósito de la incursión por la biología, Peirce expone las ideas de Darwin, Clarence King y Lamarck. De Darwin, muestra las bases de la teoría de la evolución, el factor hereditario y la conservación de las especies; esta última indica que la rata de nacimiento debe ser mayor que la de muerte. Pero desde esta perspectiva, los dos factores se presentan por variación azarosa. De Lamarck señala que en su propuesta considera que las variaciones son insensibles, porque se presentan durante la vida de los individuos, como consecuencia del esfuerzo y del ejercicio. Peirce dice que Lamarck destaca la 
importancia de los individuos, mientras que Darwin destaca la de las especies, en la medida en que considera caracteres benéficos para una raza, aunque puedan resultar fatales para los individuos: "(...) la evolución darwiniana es evolución por la acción del azar, y la destrucción de malos resultados, mientras que la evolución lamarckiana es evolución por efecto del hábito y del esfuerzo" (C.P. 6.16). Clarence King, contrariamente a Darwin y Lamarck, considera que la evolución se presenta de manera externa a los individuos o a las especies; éstos cambian, porque hay cambios en el medio ambiente. Para él, en circunstancias ordinarias no hay cambio o si lo hay es muy leve; el cambio considerable se presenta después de cataclismos: "Bajo circunstancias nuevas, nosotros vemos que animales y plantas tienden a transformarse (...) "(C.P. 6.17). Por ahora, se puede decir que el sistema cerrado que ofrece la física moderna, se tiene que abrir en el momento en que la noción general de ley vuelva a aterrizar en ella.

Luego, Peirce pasa a la psicología; allí se pueden considerar los fenómenos propios de la mente bajo tres categorías; primera: la sensación; segunda: el sentido de reacción y tercera: las concepciones generales. "Las sensaciones incluyen todo lo que es inmediatamente presente, tal como el dolor, el azul, la alegría (...). Una sensación es un estado mental (...) independiente de cualquier otro estado de la mente (...) Una sensación es simple en sí misma" (C.P. 6.1 8). En la segunda categoría se tiene que, ante un estímulo cualquiera, siempre se va a presentar una respuesta, una reacción, que existe en un aquí y un ahora. El sentido de reacción tiene dos tipos, uno interno y otro externo: "puede ser una percepción de relación entre dos ideas, o puede ser un sentido de acción y reacción entre una sensación y algo fuera de una sensación" (C.P. 6.1 9). El sentido de reacción externa también tiene dos formas, el sentido de algo que nos ha sucedido y un sentido de resistencia. El sentido de reacción considera conexiones y comparaciones.

De las concepciones generales dice: "Cuando nosotros pensamos somos conscientes que una conexión entre sensaciones es determinada por una regla general (...) que está gobernada por un hábito" (C.P. 6.20). Lo que decir que llegamos a las concepciones generales o a las leyes, una vez se ha captado en la conciencia ciertas regularidades. Esto se entenderá mejor cuando se hable de las tres categorías metafísicas. Por ahora, se puede considerar que en la psicología la noción de ley está asociada con la categoría de las concepciones generales, y ella a su vez, involucra las otras dos, la de sensación y la del sentido de reacción. Y reitera una noción que ya se había considerado en la biología, el hábito. Las ideas que se han considerado tanto en la biología como en la psicología, muestran la necesidad de ampliar la exactitud y el determinismo que creen tener las leyes mecánicas. Se debe hablar en términos de desarrollo, pues el determinismo y la exactitud cristalizarían el pensamiento y no habría lugar para la formación de nuevos hábitos: "La ley de la mente sólo hace de una sensación dada algo más probable para surgir. Esto se parece a las fuerzas no conservativas" de la física, tales como la viscosidad, la probabilidad, que son debidas a uniformidades estadísticas en el choque azaroso de trillones de partículas" (C.P. 6.23$)^{5}$.

La siguiente ciencia por la que pasa Peirce es la tradición filosófica, la cual llama idealismo objetivo. Peirce señala que, debido al dualismo cartesiano, las leyes de la materia y las leyes de la mente son radicalmente diferentes, en la medida en que cada una habla de una sustancia diferente a la otra. Cuando las leyes psicológicas derivan de las leyes físicas, se habla de un materialismo y cuando las leyes físicas derivan de las

\footnotetext{
${ }^{5}$ Causa gran curiosidad que Peirce, sabiendo esto, no lo Introdujera en la consideración de ley en el paso por la física, sino que sólo se limitó a mostrar las falencias de las leyes mecánicas, y a mostrar por qué no seria cierto que ellas pudieran explicar todos los fenómenos que ocurren en el mundo.
} 
leyes psicológicas, se habla de un idealismo. La consideración que hace Peirce aquí, es llamar la atención en la dificultad del dualismo cartesiano, y abonar el terreno para su propuesta monista, que la hace en otro de los artículos de esta serie.

En el paso parlas matemáticas, Peirce hace dos consideraciones, primera: "Los axiomas metafísicos son imitaciones de los axiomas geométricos (...)" (C.P. 6.29). La segunda, que se puede considerar como un corolario de la primera: es la importancia de tener una idea amplia de espacio, pues ésta influye en la consideración de los conceptos generales. La perspectiva es un ejemplo de la creatividad matemática para representar más de lo que de hecho se da, pues gracias a la perspectiva, se da la impresión de tres dimensiones, cuando realmente se trabaja en dos dimensiones. En este caso se toman unas líneas que representan planos a diferentes distancias y unos rayos que van desde un origen escogido hacia diversos puntos de las líneas iniciales: "Además, de acuerdo a este modo de representación todo punto de un plano representa a un punto en el otro plano" (OP. 6.26). Hay puntos de un plano que no representan puntos del otro plano, sino que se representan a sí mismos. Para un pintor que recurre a la perspectiva, estos casos son excepciones, los matemáticos no usan el término "excepción"; bajo cualquier circunstancia, ellos buscan la generalización. A su vez, la cuestión de la perspectiva, trae implícita la cuestión de la medida, asignándole un número a cada punto, pero hay puntos a los cuales no se les puede asignar un número.

De las hipótesis que se pueden manejar de espacio, Peirce considera tres: cuando el espacio es ilimitado e inmensurable (geometría euclidiana); cuando el espacio es inmensurable y limitado (geometría de Gauss) y cuando el espacio es finito e ilimitado (geometría euclidiana). De las tres hipótesis, ¿cuál sería la verdadera? La que esté influyendo en la mente: "y esto no da el más leve indicio de considerarlas exactas" (OP. 6.29). Esta última pregunta no es una estocada contra la concepción de verdad, pero sí contra la concepción de verdad absoluta y exacta, ya que si se considera el conocimiento como un entramado de relaciones, no habrá una verdad absoluta que dé cuenta de todo, sino que habrá un conjunto de verdades considerado desde diferentes puntos de vista.

Peirce vuelve a la filosofía, para hablar de tres categorías metafísicas, como una aplicación de la lógica a la filosofía: "Ellas son concepciones muy amplias y consecuentemente indefinidas (...) Primero, es la condición de ser o existir independiente de algún otro. Segundo, es la concepción de ser relativo a, la concepción de reacción con algún otro. Tercero, es la concepción de mediación, parlo cual un primero y un segundo son llevados en una relación (...). En psicología sensación es primero, sentido de reacción segundo y concepción general tercero" (OP. 6.32). A partir de las tres categorías se puede entender mejor el sentido del análisis en la psicología, y por supuesto al examen que se propuso inicialmente, pues si bien es cierto que Peirce da inicio a su examen para buscar, cuáles eran los materiales adecuados para reconstruir la filosofía, se puede hacer uso de ese análisis para otros fines diferentes a los que se propusieron.

Respecto al interés peirceano, él sostiene: "Tales son los materiales para que una teoría filosófica deba ser construida, en orden a representar el estado del conocimiento que el siglo XIX nos ha traído (...) nosotros podemos prever qué clase de metafísica sería apropiada para que sean construidas tales concepciones" (OP. 6.33). Los materiales adecuados son el concepto de ley, azar, evolución, hábito, categorías metafísicas y categorías aplicadas, continuidad y método sintético, entre otros. El método sintético es el recogimiento de las 'ideas valiosas' en el paso parlas diferentes ciencias, para obtener conceptos generales y flexibles una vez se vuelva a la filosofía. Así mismo, se puede 
volver a considerar la noción de universalidad, pero en un sentido dinámico; es decir, que considere los sistemas en constante evolución.

En resumen, para adquirir un sistema evolutivo, sólido y armonioso en la filosofía, y no sólo un sistema de ideas interesantes, pero accidentales, Peirce aconseja realizar un examen del conocimiento humano. Pero, ¿qué entendemos por conocimiento humano? Mucho se ha escrito acerca de ello; aquí nos centramos en la consideración peirceana, la cual se puede parafrasear de la siguiente manera. El mundo es un complejo compuesto de elementos, conceptos y una serie de relaciones entre unos y otros, e inclusive, entre las mismas relaciones. Su explicación implica orden y estratificación, por lo que las teorías se pueden considerar como maneras de explicar el mundo. Estas teorías buscan una relación adecuada y convincente entre leyes naturales y leyes construidas ${ }^{6}$. Para explicar el mundo, se ve la importancia de establecer una división de las ciencias y un recorrido por algunas de ellas, pues, para el propósito de la arquitectónica, el examen por una sola ciencia no resultaría ser satisfactorio, porque una idea general no se puede reducir a una localidad. Entonces, el recorrido arranca con la dinámica y con la idea de 'ley de conservación de la energía', pero, su consideración es rígida y local, pues es válida para un pequeño dominio. En el paso por la biología, la psicología, las matemáticas y la filosofía, se observa que en ellas hay elementos que suplen las falencias de las leyes mecánicas, como la consideración de la evolución, el hábito y las categorías peirceanas.

Una vez que se ha presentado la propuesta peirceana de "La arquitectura de las teorías", se puede decir que es importante establecer distinciones entre las diferentes ciencias, pero no se debe alimentar este interés hasta últimas consecuencias, porque se estarían construyendo ciencias autónomas e independientes, a tal punto que quedarían cerradas e incomunicadas entre ellas mismas. Es decir, es necesario que cada ciencia determine su objeto de estudio; un método, sin que este se constituya en el método por excelencia; y establecer unos conceptos, relaciones y problemas propios, que le den un carácter particular. A su vez se deben buscar canales de comunicación. Un caso evidente lo tenemos ahora mismo con la física, la biología y la informática; son ciencias con características propias, cuyo concepto común es el de información, y un problema común, la búsqueda de métodos adecuados para transformar códigos a un cierto lenguaje. En otras palabras, en las ciencias, hay puntos nodales que tornan evidente la necesidad de comunicación entre ellas, para analizar cómo cada una le da solución al problema, en el que todas tienen puesto su interés, y ver si ese método da luces para contextual izarlo en otra de ellas. O ver si hay elementos útiles si no se trata del método mismo. Este trabajo favorece al científico y al epistemólogo o filósofo de la ciencia, en su interés por saber en qué consiste el conocimiento humano.

En las diferentes ciencias, aunque existan características particulares, se deben buscar los conceptos comunes, para compararlos y ampliar su definición particular, con la intención de hacerlos extensivos a otros dominios, hasta ahora inexplorados, que pueden ayudar a describir y explicar un conocimiento en constate evolución.

El concepto que aquí se analizó fue el de ley El concepto de ley de la física newtoniana fue el modelo a seguir, pero por diversas circunstancias, los científicos se dieron cuenta que estas leyes no podían explicar todos los fenómenos. Esto no quiere decir que las leyes de la física clásica no sean válidas, sino que lo son, en un terreno restringido. De la misma manera hay otras leyes que explican otros dominios y no describen los fenómenos

\footnotetext{
${ }^{6}$ A propósito del realismo peirceano, no habría mayor distinción entre las leyes naturales y las leyes construidas, las leyes naturales son las que están allí donde el científico está buscando, y las leyes construidas serian esas mismas leyes naturales expresadas en un lenguaje comen para dicha comunidad de científicos.
} 
clásicos; es decir, no se encontrará una única ley que dé cuenta de todos los fenómenos, se encontrarán conjuntos de leyes, que en algún momento, se encontrarán en espacios comunes con leyes de otras ciencias, o con ramas de la misma ciencia.

¿De qué manera se conecta parte de la propuesta peirceana, con el propósito del artículo, de resquebrajar ciertos prejuicios cognitivos? Se parte de la tesis de que se necesitan unos principios generales para solucionar los problemas y describir fenómenos propios de una época, desde diferentes puntos de vista. Entonces, el problema a solucionar en este artículo es, ¿cómo destruir prejuicios cognitivos que impiden el desarrollo del pensamiento? La solución se puede encontrar en la propuesta de Peirce. Los prejuicios cognitivos se empiezan a formar cuando, primero, se pretende explicar la totalidad del mundo; segundo, cuando una explicación local se quiere aplicar a todo. La física newtoniana, como sistema cognitivo, pretendió explicar absolutamente todos los fenómenos, pero sus principios, tanto matemáticos, lógicos, físicos y metafísicos, sirvieron para dar cuenta de una pequeña cantidad de fenómenos. Los principios de una época no son los mismos de otra, inclusive se podría decir que la diferencia de una época y otra, está dada parlas principios que la rigen. No se trata de echarle el agua sucia a la física clásica, porque ella cumplió con su papel en la modernidad, pero lo que no se quiere es perpetuar su estructura, por útil que haya sido.

Respecto a la interdisciplinariedad que señala Peirce, se quiere ir más allá diciendo que la interdisciplinariedad no es más que el reflejo de unos principios abordados desde diferentes puntos de vista, los cuales no son otros que las diferentes ciencias, y no es que una de ellas se convierta en el paradigma o modelo a seguir de las otras, sino que es la que mejor logra plasmar tales principios. En la modernidad, la que mejor lo hizo fue la física, aunque las demás ciencias estuvieran haciendo lo propio. Tales principios fueron: la causalidad, la reversibilidad en el tiempo, el equilibrio y la diferenciabilidad de los fenómenos; el espacio que se utilizó fue el euclidiano, y la base lógica, la dualidad verdadero-falso. Este esquema estuvo en la biología, la química y la sociología, entre otras, y caracterizó el siglo XVII, XVIII y gran parte del XIX, sólo que aquí, el paso de la doxa a la episteme no es gradual y lineal, sino que es un constante devenir de la una a la otra. El sentido común va más lento, es propio de la intuición y de la mayoría de personas. Lo que quiere decir, que el conocimiento no avanza de manera homogénea; parlo tanto, sus estrategias no son las mismas, y los prejuicios, sean unos o sean otros, siempre se van a presentar. Hacerle frente al pensamiento moderno fue muy difícil; se empezó a gestar a finales del siglo XIX, pero aún hoy, como cultura, no lo hemos digerido completamente, y sin duda alguna no lo llegaremos a hacer.

Hoy en día, la biología, la termodinámica, la informática, la semiótica, etc., nos muestran la necesidad de consolidar otros principios, y aquí se vuelve a retomar a Peirce pues esos serían aquellos en los que él tanto insistió en "La arquitectura de las teorías", a evolución, la continuidad y el azar. Aquí, como en la modernidad, hay una ciencia que se destaca entre las demás, la biología ${ }^{7}$. Las certezas y los términos absolutos han quedado restringidos a un pequeño dominio del saber; ahora lo que despierta el interés en las ciencias es la información y cómo pasar unos códigos desconocidos a un cierto lenguaje. Pero estos principios en algún momento quedarán confinados a un pequeño rincón del conocimiento, para darle lugar a otros, y así sucesivamente, de manera que se tienda, pero no se alcance la perfección.

\footnotetext{
${ }^{7}$ Al decir que la biología es la ciencia que se destaca, solo se quiere decir que, entre las ciencias, es la que mejor logra plasmar las características de esta época.
} 


\section{BIBLIOGRAFÍA}

Andrade, E. Los demonios de Darwin. Bogotá, Unibiblos, 2000.

Apel, K. El camino del pensamiento de Charles S. Peirce. Madrid, Visor, 1997.

Asimov, I. Grandes ideas de la ciencia. Madrid, Alianza, 1989.

Boltzmann, L. Escritos de mecánica y termodinámica. Madrid, Alianza, 1986.

Kuhn, T. S. La estructura de las revoluciones científicas. México, Fondo de Cultura Económica, 1986.

. ¿Qué son las revoluciones cien tíficas? Y otros ensayos. Barcelona, Altaya, 1995.

Newton-Smith, W. H. La racionalidad de la ciencia. Barcelona, Paidós, 1981.

Ortoli, S., Pharabod, J. P. El cántico de la cuántica. Barcelona, Gedisa, 1987.

Peirce, Ch. S. Collected Papers, 8 vols. (Hartshorne, Weiss, Burks eds.), Cambridge, Harvard University Press, 1931-1958.

"Diálogos", la doctrina de la necesidad examinada, traducción de Georges Delacre. San Juan, Libros de México, 1973.

. Escritos lógicos. Madrid, Alianza Universidad, 1968.

'A Guess at the Riddle' (o. 1888), en: Charles S. Peirce, The Essential Peírce. Selected Philosophical Writings (eds. Houser, Kloesel). Bloomington, Indiana University Press, 1992.

. "La ley de la mente, El hombre, un signo. Barcelona, Crítica, 1988.

. The New Elements of Mathematics (ed. Carolyn Eisele). La Haya, Mouton, 1976

. Reasoning and the Logic of Things (eds. K. L. Ketner, H. Putnam). Cambridge, Harvard University Press, 1992.

v.4,1982-1993.

Writings (A Chronological Edítion). Bloomington, Indiana University Press,

Popper, K. Teoría cuántica y cisma en física. Madrid, Tecnos, 1985.

Rae, A., Física cuántica, ¿ilusión o realidad? Madrid, Alianza, 1986. 
